\title{
Template Matching based Eye Detection in Facial Image
}

\author{
Nilamani Bhoi \\ Institute of Technical Education \& Research \\ Siksha 'O' Anusandhan University \\ Bhubaneswar, India
}

\author{
Mihir Narayan Mohanty* \\ Institute of Technical Education \& Research \\ Siksha 'O' Anusandhan University \\ Bhubaneswar, India
}

\begin{abstract}
Eye detection is a pre-requisite stage for many applications such as human-computer interfaces, iris recognition, driver drowsiness detection, security, and biology systems. In this paper, template based eye detection is described. The template is correlated with different regions of the face image. The region of face which gives maximum correlation with template refers to eye region. The method is simple and easy to implement. The effectiveness of the method is demonstrated in both the cases like open eye as well as closed eye through various simulation results.
\end{abstract}

\section{Keywords}

Eye detection, template matching, Cross-correlation, pattern recognition

\section{INTRODUCTION}

Human face image analysis, detection and recognition have become some of the most important research topics in the field of computer vision and pattern classification. Recognition of human faces out of still images or image sequences is an actively developing research field. In recent years, new technology begins to emerge in the field of computer engineering and this gives rise to other methods of human-computer interaction such as through voice and eye-gaze. Various researches on human-computer interaction or other types of application based on eye gaze have been done. Apart from it, eye detection is a crucial aspect in many useful applications ranging from face recognition and face detection to human computer interface design, driver behavior analysis, and compression techniques development. By locating the position of the eyes, the gaze can be determined. A large number of works have been published in the last decade on this subject. Generally the detection of eyes is done in two steps: locating face to extract eye regions and then eye detection from eye window.

Recently, methods based on boosting have become the focus of active research. The eye detection is done in the face regions which have been already located [1, 2, 3, 4]. Little research has been done, however, on the direct search for eyes in whole images. In [1], iris geometrical information is used for determining a region candidate that contains an eye in the whole image, and then the symmetry is used for selecting the pair of eyes.

Although the detection rate is high, the assumption that the distance between the camera and the person does not change greatly limits its practical applicability. The main objective of this work is to propose an eye detection algorithm that is applicable with a standard camera, in a real world condition, while skipping the initial segmentation step to extract the face region as commonly done in literature. The developed eye detection algorithm works on the whole grey-level image.

\section{LITERATURE REVIEW}

A lot of research work has been published in the field of eye detection in the last decade. Various techniques have been proposed using texture, depth, shape and color information or combinations of these for eye detection. Vezhnevets et al. [5] focus on several landmark points (eye corners, iris border points), from which the approximate eyelid contours are estimated. The iris center and radius is detected by looking for a circle separating dark iris and bright sclera. The upper eyelid points are found using on the observation that eye border pixels are significantly darker than surrounding skin and sclera. The detected eye boundary points are filtered to remove outliers and a polynomial curve is fitted to the remaining boundary points. The lower lid is estimated from the known iris and eye corners. Reinders et al. [6] present a method where based on the technique of template matching the positions of the eyes on the face image can be followed throughout a sequence of video images. To increase the robustness of the tracking scheme the method automatically generates a codebook of images representing the encountered different appearances of the eyes. Yuille et al. [7] first proposed using deformable templates in locating human eye. The weaknesses of the deformable templates are that the processing time is lengthy and success relies on the initial position of the template. Lam et al. [8] introduced the concept of eye corners to improve the deformable template approach. Saber et al. [9] and Jeng et al. [10] proposed to use facial features geometrical structure to estimate the location of eyes. Takacs et al. [11] developed iconic filter banks for detecting facial landmarks. Feng and Yeun [12] developed a variance projection function for locating the corner points of the eye. Zhou and Geng [13] propose a hybrid projection function to locate the eyes. By combining an integral projection function, which considers mean of intensity, and a variance projection function, which considers the variance of intensity, the hybrid function better captures the vertical variation in intensity of the eyes. Kumar et al. [14] suggest a technique in which possible eye areas are localized using a simple thresholding in color space followed by a connected component analysis to quantify spatially connected regions and further reduce the search space to determine the contending eye pair windows. Finally the mean and variance projection functions are utilized in each eye pair window to validate the presence of the eye. Feng and Yeun [15] employ multi cues for eye detection on gray images using variance projection function. Various other methods that have been adopted for eye detection include wavelets, principal component analysis, fuzzy logic, support vector machines, neural networks, evolutionary computation and hidden 
markov models. Huang and Wechsler [16] perform the task of eye detection by using optimal wavelet packets for eye representation and radial basis functions for subsequent classification of facial areas into eye and non-eye regions. Filters based on Gabor wavelets to detect eyes in gray level images are used in [17]. In $[18,19]$ eigen eyes are calculated by applying Karhunen-Loeve Transformation to represent the major characteristics of human eyes and are stored as reference patterns for the localization of human eyes in video images. Given a new input image of the same size, it is projected into the eigeneye space. The produced vector describes the similarity of this new image to the eigeneyes. If similarity measure (the Euclidean distance between the mean adjusted input image and its projection onto the eigeneye space) smaller than a threshold, the new image is classified as an eye region. All these methods have more computational complexity as well as implementation difficulty for hardware.

\section{METHODOLOGY}

One of the popular method is template matching. In this method, an eye template $T$ is used to detect the eye from face image. The correlation of eye template with various overlapping regions of the face image is found out. The region with maximum correlation with the template refers to eye region. The block diagram of the method is shown in Fig.1.

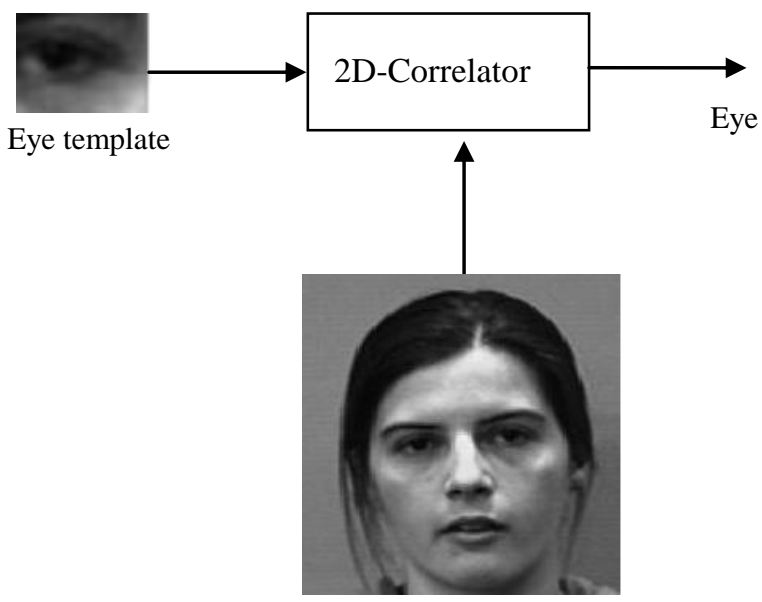

Face Image

Fig.1. Template matching based eye detection

\subsection{Template Matching}

Let I be an image of dimension $m \times n$ and $T$ be another image of dimension $p \times q$. Template matching can be defined as a search method which finds out the portion in $I$ of size $p \times q$ where $T$ has the maximum cross correlation coefficient with it.

\subsubsection{Cross-correlation Coeficient}

The cross correlation coefficient is defined as

$$
\gamma(x, y)=\frac{\sum_{s} \sum_{t} \delta_{I(x+s, y+t)} \delta_{T(s, t)}}{\sum_{s} \sum_{t} \delta_{I(x+s, y+t)}^{2} \delta_{T(s, t)}^{2}}
$$

Where,

$$
\begin{aligned}
& \delta_{I(x+s, y+t)}=I(x+s, y+t)-\bar{I}(x, y), \\
& \delta_{T(s, t)}=T(s, t)-\bar{T} \\
& s \in\{1,2,3, \ldots . ., p\} \\
& t \in\{1,2,3, \ldots . ., q\}, \\
& x \in\{1,2,3, \ldots ., m-p+1\}, \\
& y \in\{1,2,3, \ldots . ., n-q+1\}, \\
& \bar{I}(x, y)=\frac{1}{p q} \sum_{s} \sum_{t} I(x+s, y+t) \\
& \bar{T}=\frac{1}{p q} \sum_{s} \sum_{t} T(s, t)
\end{aligned}
$$

The value of cross-correlation coefficient $\gamma$ ranges from $-l$ to $+l$ corresponds completely not matched and completely matched respectively. For template matching the template, $T$ slides over $I$ and $\gamma$ is calculated for each coordinate $(x, y)$. After calculation, the point which exhibits maximum $\gamma$ is referred to as the match point.

\subsubsection{The Algorithm:}

The method of template matching is given as an algorithm, which is so simple and easy to implement. The algorithm steps are as follows :

Step 1: An eye template of size $m \times n$ is taken.

Step 2: The normalized 2-D auto-correlation of eye template is found out.

Step 3: the normalized 2-D cross-correlation of eye template with various overlapping regions of the face image is calculated.

Step 4: The mean squared error (MSE) of auto correlation and cross-correlation of different regions are found out. The minimum MSE is found out and stored.

Step 5: the region of the face corresponding to minimum MSE represents eye region.

\section{RESULT}

The proposed method is implemented in the MALAB 7.0 platform. All the images used for the purpose of simulation are of sizes $512 \times 512$. The algorithm is tested on the face images having the eyes on both states [open and closed]. 

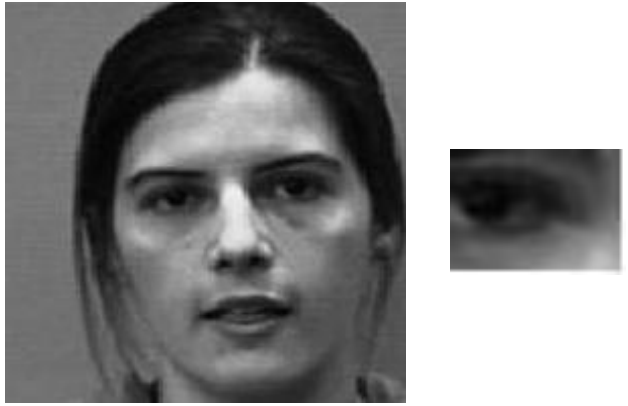

(a)
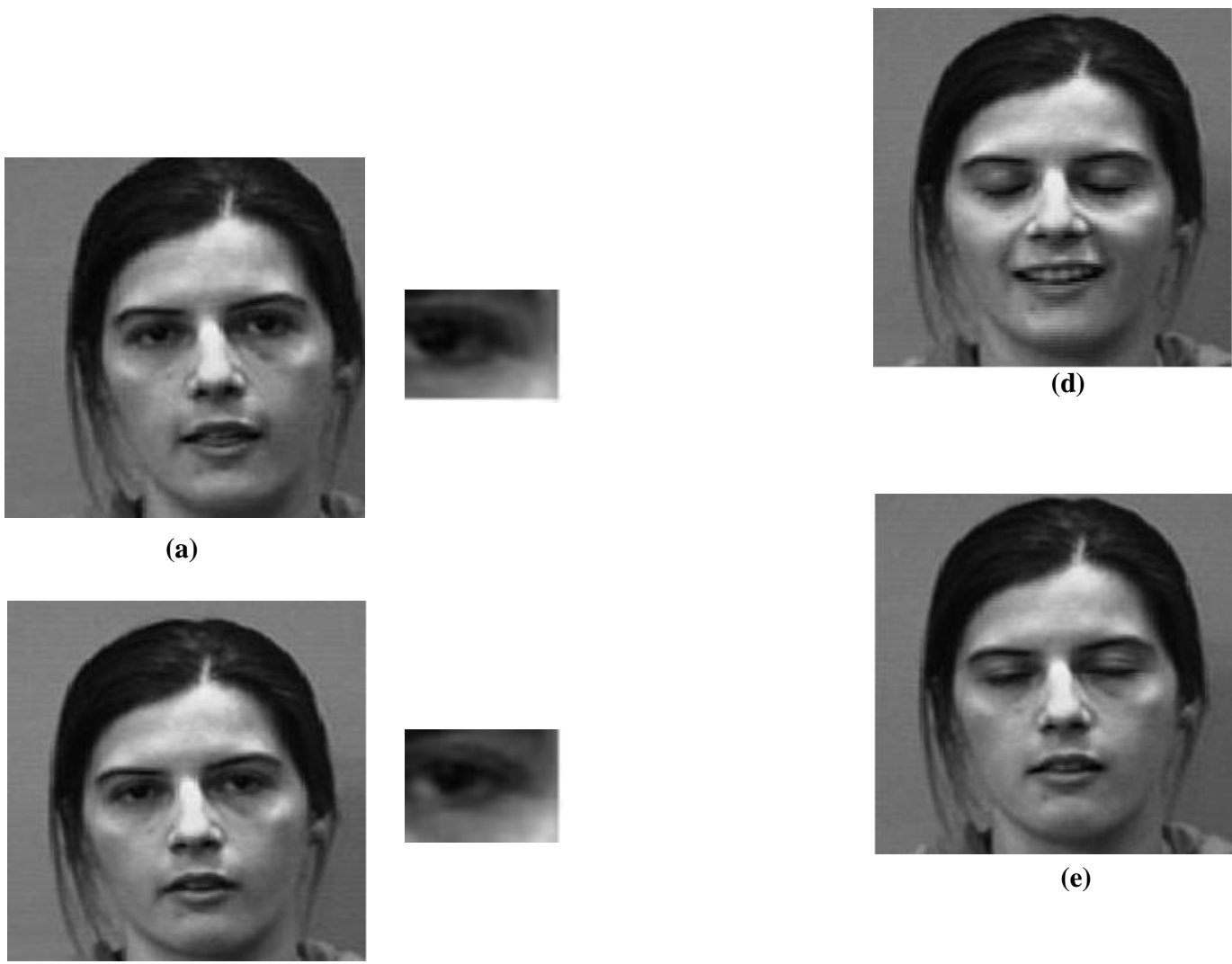

(d)

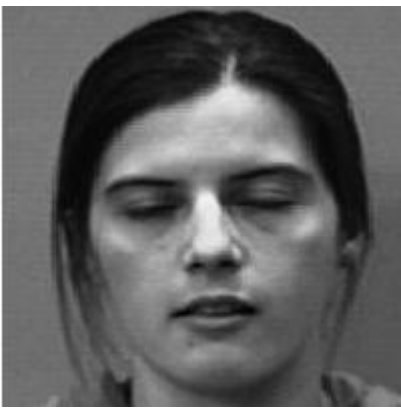

(e)

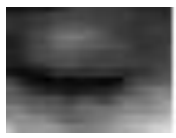

(b)

Fig. 2. (a) - (e) face images ; to the right zoomed version of eyes extracted from face. 


\section{CONCLUSION}

A novel and simple eye detection scheme is proposed in this paper. An eye template is used to detect eye region from face image. The template is matched with eye region using cross correlation technique. The method does not require any complex mathematical calculation and prior knowledge about the eye. It is a simple method and can easily be implemented by hardware.

\section{REFERENCES}

[1] Kawaguchi T. and Rizon M., "Iris detection using intensity and edge information," Pattern Recognition, vol. 36, pp. 549-562, 2003.

[2] Baskan S., Bulut M., and Atalay V., "Projection based method for segmentation of human face and its evaluation," Pattern Recognition Letters, vol. 23, pp.1623-1629, 2002.

[3] Sirohey S., Rosenfiled A., and Duric Z., "A method of detection and tracking iris and eyelids in video," Pattern Recognition, vol. 35, pp. 1389-1401, 2002.

[4] D'Orazio T., Leo M., Cicirelli G., and Distante A., "An algorithm for real time eye detection in face images," in Proc. 17th Int. Conf. on Pattern Recognition, vol. 3, pp. 278-281, 2004.

[5] Vezhnevets V., Degtiareva A., "Robust and Accurate Eye Contour Extraction", Proc. Graphicon-2003, pp. 81-84, Moscow, Russia, September 2003.

[6] Reinders M., "Eye tracking by template matching using an automatic codebook generation scheme", Third annual conference of the Advanced School for Computing and Imaging, pp. 85-91, Heijen, The Netherlands, June 1997.

[7] Yuille A.L., Hallinan P.W., Cohen D.S., "Feature extraction from faces using deformable templates", International Journal of Computer Vision, vol. 8, no. 2 pp. 99-111, 1992.

[8] Lam K.M., Yan H., "Locating and extracting the eye in human face images", Pattern Recognition, vol. 29, no. 5 pp.771-779, 1996.
[9] Saber E. and Tekalp A.M., "Frontal-view face detection and facial feature extraction using color, shape and symmetry based cost functions", Pattern Recognition Letters, vol. 19, no. 8, pp. 669--680, 1998.

[10] Jeng, S.H., Liao, H.Y.M., Han, C.C., Chern, M.Y., Liu, Y.T., "Facial Feature Detection Using Geometrical Face Model: An Efficient Approach", Pattern Recognition, vol. 31, no. 3, pp. 273-282, 1998.

[11] Takacs, B., Wechsler, H., "Detection of faces and facial landmarks using iconic filter banks", Pattern Recognition, vol. 30, no. 10, pp. 1623-1636, 1997.

[12] Feng, G.C., Yuen, P.C., "Variance Projection Function and its Application to Eye Detection for Human Face Recognition”, Pattern Recognition Letters, vol. 19, pp. 899. 906, 1998.

[13] Zhou Z.H. and Geng X., "Projection Functions for Eye Detection", Pattern Recognition, vol. 37, no.5, pp. 10491056, 2004.

[14] Kumar, Thilak $\mathrm{R}$ and Raja, Kumar $\mathrm{S}$ and Ramakrishnan, "Eye detection using color cues and projection functions", Proceedings 2002, International Conference on Image Processing, ICIP, vol. 3, 337-340, Rochester, New York, USA, 2002.

[15] Feng G.C. and Yuen Pong C., "Multicues eye detection on gray intensity image", Pattern Recognition. vol. 34, pp. 1033-1046, 2001.

[16] Huang J. and Wechsler H., "Eye Detection Using Optimal Wavelet Packets and Radial Basis Functions (RBFs)", International Journal of Pattern Recognition and Artificial Intelligence, vol. 13 no. 7, 1999

[17] Sirohey S.A. and Rosenfeld A., "Eye detection in a face image using linear and nonlinear filters", Pattern Recognition, vol. 34, pp. 1367-1391, 2001.

[18] Pentland A., Moghaddam B., Starner T., "View-based and modular eigenspaces for face recognition", Proceedings of the IEEE International Conference on Computer Vision and Pattern Recognition, Seattle, WA, pp.84-91, 1994.

[19] Gonzalez R.C., Woods R.E., "Digital Image Processing," $2^{\text {nd }}$ ed. Prentice Hall India, 2002. 